University of New Hampshire

University of New Hampshire Scholars' Repository

Physics Scholarship

Physics

3-2014

\title{
Effect of EMIC waves on relativistic and ultrarelativistic electron populations: Ground-based and Van Allen Probes observations
}

M. Usanova

University of Alberta

A. Drozdov

University of California - Los Angeles

K. Orlova

University of California - Los Angeles

I. R. Mann

University of Alberta

Y. Y. Shprits

University of California - Los Angeles

See next page for additional authors

Follow this and additional works at: https://scholars.unh.edu/physics_facpub

Part of the Physics Commons

\section{Recommended Citation}

Usanova, M. E., et al. (2014), Effect of EMIC waves on relativistic and ultrare-lativistic electron populations: Ground-based and Van Allen Probes observations, Geophys. Res. Lett., 41, 1375-1381, doi:10.1002/2013GL059024.

This Article is brought to you for free and open access by the Physics at University of New Hampshire Scholars' Repository. It has been accepted for inclusion in Physics Scholarship by an authorized administrator of University of New Hampshire Scholars' Repository. For more information, please contact Scholarly.Communication@unh.edu. 


\section{Authors}

M. Usanova, A. Drozdov, K. Orlova, I. R. Mann, Y. Y. Shprits, M. T. Robertson, D. L. Turner, D. K. Milling, A. Kale, D. N. Baker, S. A. Thaller, Geoffrey Reeves, Harlan E. Spence, C A. Kletzing, and J. R. Wygant 


\section{Geophysical Research Letters}

\section{RESEARCH LETTER}

10.1002/2013GL059024

\section{Special Section:}

Early Results From the Van

Allen Probes

Key Points:

- EMIC wave activity is not associated with precipitation of $\mathrm{MeV}$ electrons

- EMIC waves do not deplete the ultrarelativistic belt down to $90^{\circ}$

- EMIC waves cause loss of low pitch angle electrons with energies $\sim 2-8 \mathrm{MeV}$

\section{Correspondence to:}

M. E. Usanova,

musanova@ualberta.ca

\section{Citation:}

Usanova, M. E., et al. (2014), Effect of EMIC waves on relativistic and ultrarelativistic electron populations: Groundbased and Van Allen Probes observations, Geophys. Res. Lett., 41, 1375-1381, doi:10.1002/2013GL059024.

\section{Received 7 JAN 2014} Accepted 7 FEB 2014

Accepted article online 12 FEB 2014 Published online 6 MAR 2014

\section{Effect of EMIC waves on relativistic and ultrarelativistic electron populations: Ground-based and Van Allen Probes observations}

\author{
M. E. Usanova ', A. Drozdov',3, K. Orlova 2,3 , I. R. Mann' ${ }^{\text {, Y. Shprits }}{ }^{2,4,5}$, M. T. Robertson' ${ }^{\text {, D. L. Turner }}{ }^{2}$, \\ D. K. Milling ${ }^{1}$, A. Kale ${ }^{1}$, D. N. Baker ${ }^{6}$, S. A. Thaller ${ }^{7}$, G. D. Reeves ${ }^{8}$, H. E. Spence ${ }^{9}$, C. Kletzing ${ }^{10}$, and J. Wygant ${ }^{7}$
}

${ }^{1}$ Department of Physics, University of Alberta, Edmonton, Alberta, Canada, ${ }^{2}$ Department of Earth, Planetary, and Space Sciences, University of California, Los Angeles, California, USA, ${ }^{3}$ Lomonosov Moscow State University Skobeltsyn Institute of Nuclear Physics, Moscow, Russia, ${ }^{4}$ Skolkovo Institute of Science and Technology, Skolkovo, Russia, ${ }^{5}$ Massachusetts Institute of Technology, Cambridge, Massachusetts, USA, ${ }^{6}$ Laboratory for Atmospheric and Space Physics, University of Colorado Boulder, Boulder, Colorado, USA, ${ }^{7}$ School of Physics and Astronomy, University of Minnesota, Minneapolis, Minnesota, USA, ${ }^{8}$ Los Alamos National Laboratory, Los Alamos, New Mexico, USA, ' Institute for the Study of Earth, Oceans, and Space, University of New Hampshire, Durham, New Hampshire, USA, ${ }^{10}$ Department of Physics and Astronomy, University of lowa, lowa City, lowa, USA

Abstract We study the effect of electromagnetic ion cyclotron (EMIC) waves on the loss and pitch angle scattering of relativistic and ultrarelativistic electrons during the recovery phase of a moderate geomagnetic storm on 11 October 2012. The EMIC wave activity was observed in situ on the Van Allen Probes and conjugately on the ground across the Canadian Array for Real-time Investigations of Magnetic Activity throughout an extended $18 \mathrm{~h}$ interval. However, neither enhanced precipitation of $>0.7 \mathrm{MeV}$ electrons nor reductions in Van Allen Probe $90^{\circ}$ pitch angle ultrarelativistic electron flux were observed. Computed radiation belt electron pitch angle diffusion rates demonstrate that rapid pitch angle diffusion is confined to low pitch angles and cannot reach $90^{\circ}$. For the first time, from both observational and modeling perspectives, we show evidence of EMIC waves triggering ultrarelativistic $(\sim 2-8 \mathrm{MeV})$ electron loss but which is confined to pitch angles below around $45^{\circ}$ and not affecting the core distribution.

\section{Introduction}

The Earth's outer radiation belt is populated by electrons of energies from $100 \mathrm{~s}$ of keV to several MeV, which are commonly referred to as energetic or relativistic electrons. During geomagnetic storms, outer belt fluxes exhibit irregular variations over several orders of magnitude on the timescales from minutes to days. Recent results showed that a very narrow third belt consisting of ultrarelativistic electrons with energy $>2 \mathrm{MeV}$ can be formed and exist for over a month [Baker et al., 2013]. These new observations suggest that the ultrarelativistic electron population constitutes an important part of the near-Earth environment and the processes that define its lifetime are not yet fully understood.

Electromagnetic ion cyclotron (EMIC) waves are transverse plasma waves in the frequency range between 0.1 and $5 \mathrm{~Hz}$. EMIC waves are generated in the magnetosphere by ring current protons with temperature anisotropy in three bands, below the $\mathrm{H}^{+}, \mathrm{He}^{+}$, and $\mathrm{O}^{+}$ion gyrofrequencies. EMIC wave growth can lead to the isotropization of the initially unstable proton distribution and loss of protons into the atmosphere [see, e.g., Cornwall, 1965; Usanova et al., 2010].

EMIC waves can also undergo a cyclotron resonance with radiation belt electrons and cause precipitation of electrons into the atmosphere. The resonance energy of relativistic electrons interacting with EMIC waves is very sensitive to the ratio of local plasma density to the magnetic field strength and, in the cold multi-ion plasma approximation, to the proximity of the wave frequency to ion gyrofrequencies [e.g., Summers and Thorne, 2003]. In the cold plasma approximation, EMIC waves can interact with electrons below 1-2 MeV only in regions of high plasma density [e.g., Ukhorskiy et al., 2010]. Therefore, EMIC waves are expected to have a much more pronounced effect on ultrarelativistic electron pitch angle scattering. Shprits et al. [2013] simulated the formation of the third radiation belt observed by Baker et al. [2013] and concluded that EMIC waves were a necessary component of the plasma environment required to produce loss of the ultrarelativistic electrons and hence to explain the observed narrow radiation belt configuration. In this paper, we further investigate interactions between EMIC waves and ultrarelativistic electrons from both observational and modeling perspectives. 


\section{Instrumentation}

\subsection{Magnetometer Array}

We used ground-based magnetic field measurements from the Canadian Array for Real-time Investigations of Magnetic Activity (CARISMA; www.carisma.ca) [see Mann et al., 2008]. For this study, we used data from search coil magnetometers with resolution of $<0.2 \mathrm{pT} / \mathrm{Hz}{ }^{1 / 2}$ at $1 \mathrm{~Hz}$, which span the range of L-shells between $\mathrm{L}=3.6-6$ and $\sim 4 \mathrm{~h}$ MLT.

\subsection{NOAA POES}

The National Oceanic and Atmospheric Administration (NOAA) Polar Orbiting Environmental Satellites (POES) are in circular, Sun synchronous orbits at altitude $\sim 850 \mathrm{~km}$. POES carries onboard a Medium Energy Proton and Electron Detector (MEPED) that monitors intensities of charged particle radiation [Evans and Greer, 2000]. There are two identical proton telescopes: the $0^{\circ}$ detector, monitoring particles in the atmospheric loss cone throughout outer belt latitudes and the $90^{\circ}$ detector, monitoring particles that are mirroring near the satellite. We used data from the $30-80 \mathrm{keV}, 80-250 \mathrm{keV}, 250-800 \mathrm{keV}$, and above $6900 \mathrm{keV}$ proton channels sampled at 0.5 samples/s. Since the last channel is also sensitive to $>0.7 \mathrm{MeV}$ electrons [e.g., Rodger et al., 2010], we used it to monitor precipitation of relativistic electrons.

\subsection{Van Allen Probes}

The Van Allen Probes are two identical spacecraft in similar orbits and encompassing both the inner and outer radiation belts [Kessell et al., 2012]. Their highly elliptical orbits range from a minimum altitude of $\sim 600 \mathrm{~km}$ to a maximum altitude of $\sim 37,000 \mathrm{~km}$. To look at the dynamics of the ultrarelativistic electron population, we used electron flux data from the Relativistic Electron Proton Telescope (REPT), within the Energetic particle Composition and Thermal (ECT) suite [Spence et al., 2013]. REPT measures electrons with energies between $\sim 2$ and $10 \mathrm{MeV}$ [Baker et al., 2012]. For EMIC wave identification, we used 64 samples/s magnetic field measurements with accuracy $\sim 0.1 \mathrm{nT}$ from the Electric and Magnetic Field Instrument Suite and Integrated Science (EMFISIS) [Kletzing et al., 2013]. To obtain information about background cold plasma densities, we used spacecraft potential measurements from four spin-plane antennae of the Electric Field and Waves Instrument (EFW) [Wygant et al., 2013].

\section{Observations}

\subsection{Long-Lasting EMIC Wave Event on 11 October 2012}

We examine a long-lasting EMIC wave event from 11 October 2012, which occurred in the recovery of a moderate geomagnetic storm (with minimum Dst $=-111 \mathrm{nT}$ on 9 October). Continuous EMIC activity was observed on the ground by multiple CARISMA stations for more than $18 \mathrm{~h}$ (see accompanying paper by Mann et al. [2014] for more details).

The Van Allen Probes A and B were conjugate to the region of EMIC activity: at 14:15 UT, probes A and B had magnetic footprints close to the CARISMA Pinawa (PINA) and Dawson (DAWS) stations, respectively (see Figure 1). EMIC wave dynamic spectra from these two stations are shown in Figures $1 a$ and $1 b$, and the spectrogram of the Bx GSM magnetic field component on probe $B$ is shown in Figure 1c. The black line superposed on the probe $B$ spectrogram shows the local helium gyrofrequency, and the red line shows the background electron density. Similar to observations by Usanova et al. [2008], helium-band EMIC wave activity on probe B is seen on a narrow range of $L$-shells from $L \sim 4.0$ to 4.5 and is bounded at high $L$ by a density drop, which indicates the plasmapause or the plasmasphere boundary layer.

During the interval of EMIC activity on the ground, POES 16 observed a loss of energetic protons (a signature of EMIC instability) in low-Earth orbit. For example, at 14:08 UT, POES 16 registered a localized enhancement of precipitating protons with energies $>30 \mathrm{keV}$ close to PINA $(\mathrm{L} \sim 4)$. Figures $1 \mathrm{~d}-1 \mathrm{~g}$ show POES proton data from 30 to $80 \mathrm{keV}, 80$ to $250 \mathrm{keV}$, and 250 to $800 \mathrm{keV}$, and the channel sensitive to $>0.7 \mathrm{MeV}$ electrons, respectively. The blue (red) trace in Figures $1 \mathrm{~d}-1 \mathrm{~g}$ shows the flux of precipitating (locally mirroring, trapped) particles observed by MEPED. Clearly, the precipitation of energetic protons associated with the narrow Lrange of EMIC waves is not accompanied by precipitating $>0.7 \mathrm{MeV}$ energetic electrons. 

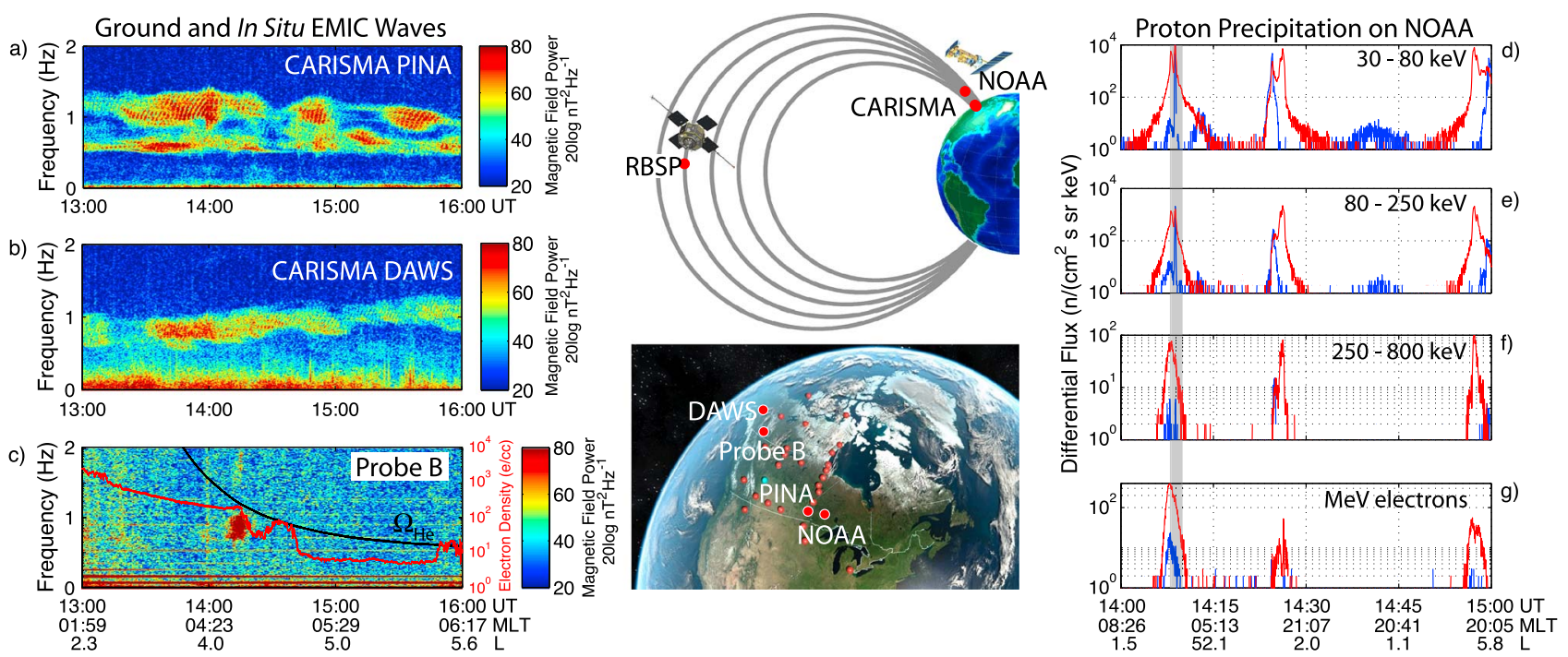

Figure 1. EMIC wave observations from the CARISMA (a) PINA and (b) DAWS magnetometers, from (c) GSM Bx component from Van Allen Probe B, (d-f) together with loss of energetic protons on the POES 16 satellite on 11 October 2012. (g) On POES 16, >0.7 MeV electron loss was not observed at the same time. The central panel shows the schematic locations of the satellites and ground magnetometers.

\subsection{POES Precipitation Observations}

To examine the global impact of EMIC waves on Van Allen belt dynamics, we extended our analysis of POES data to a longer interval (9-20 October), encompassing the 11 October 2012 event. Similar to Figure 1g, Figure 2 shows locally mirroring (trapped; Figure $2 \mathrm{a}$ ) and precipitating (Figure $2 \mathrm{~b}$ ) $>0.7 \mathrm{MeV}$ electron flux on POES (averaged over all six POES satellites) as a function of $L$ and time for this interval. The POES observations indicate that the outer radiation belt electron flux was enhanced following the 9 October 2012 storm. While POES shows that there is an appreciable amount of $>0.7 \mathrm{MeV}$ electron precipitation from the heart of the outer belt, it remains almost constant in time over this interval and is less than $5 \%$ of the trapped flux at the

a)
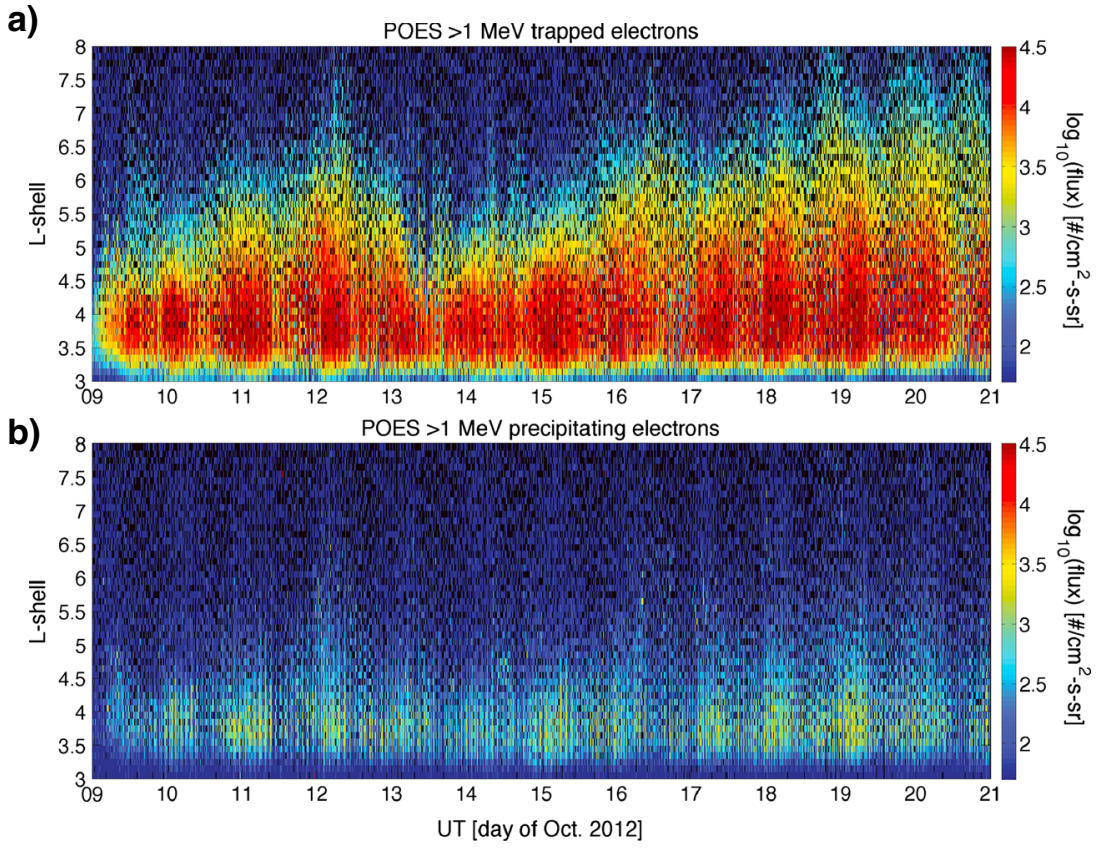

Figure 2. On POES, $>0.7 \mathrm{MeV}$ electron unidirectional differential flux (a) trapped and (b) precipitating as a function of $\mathrm{L}$ between 9 and 20 October 2012. A decrease in the electron flux occurred on 13 October during the minimum Dst (-101 nT). 


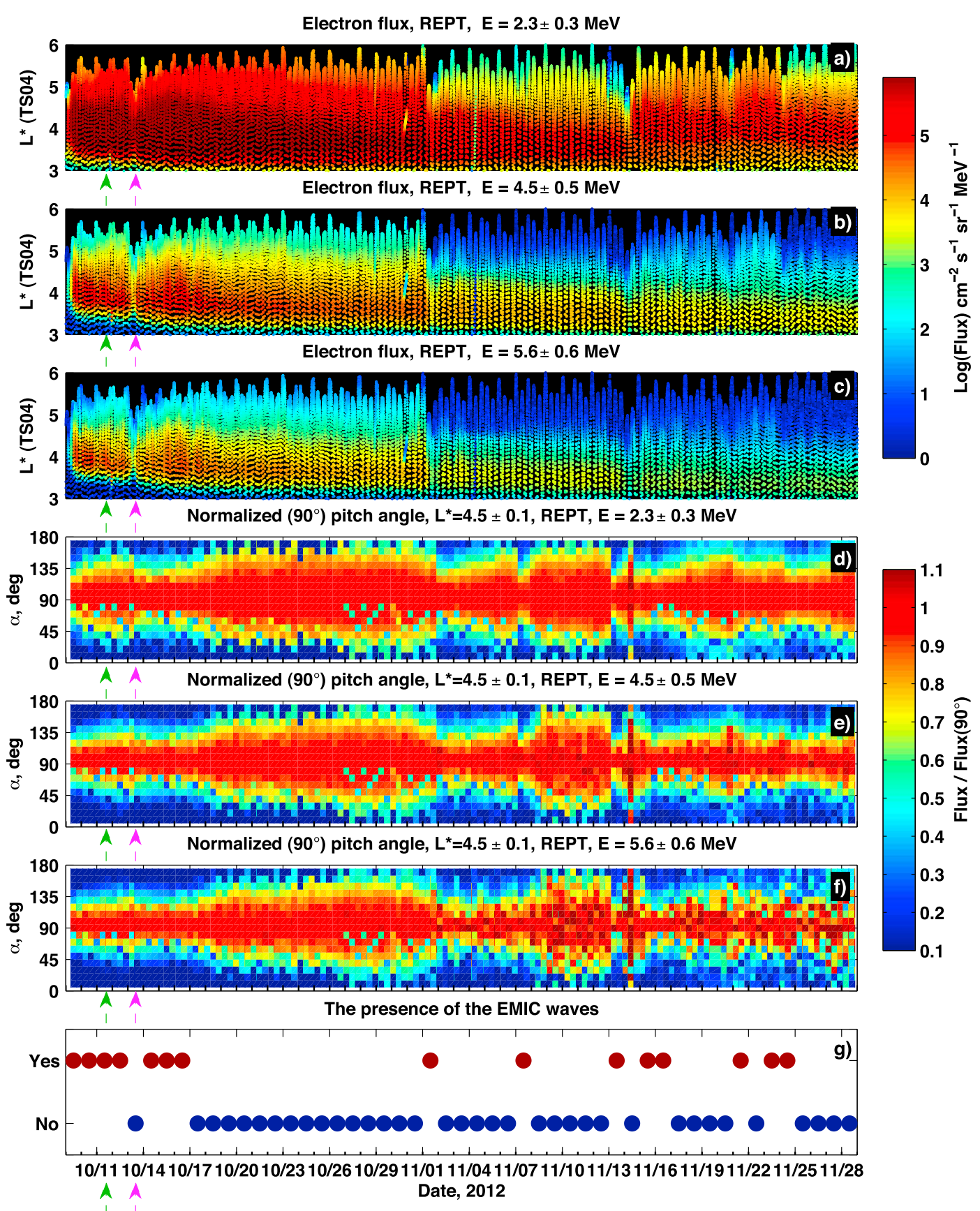

Figure 3. Omnidirectional (a-c) differential electron flux as a function of $L^{*}$ and $(d-f)$ differential flux as a function of pitch angle, normalized by the $90^{\circ}$ pitch angle flux, at $L^{*}=4.5$ in the $2.3,4.5$, and $5.6 \mathrm{MeV}$ energy channels, respectively, from REPT, and (g) EMIC wave occurrence from $L \sim 4-4.5$ on the ground between 9 October and 29 November 2012. The green arrow shows the EMIC event on 11 October. The purple arrow indicates the time of the minimum Dst at 11 UT on 13 October 2012.

edge of the loss cone. Moreover, the most significant loss of trapped flux observed on 13 October is not accompanied by either enhanced precipitation or by EMIC waves. Overall, these observations suggest that either there is little impact on the level of trapped flux arising from EMIC wave scattering of $>0.7 \mathrm{MeV}$ electrons into the loss cone or that source and/or transport dominated over any EMIC losses on 11 October.

\subsection{Ultrarelativistic Electron Observations From October to November 2012}

To continue investigating the effect of the observed EMIC wave activity on the ultrarelativistic electron flux, we used data from the REPT instruments onboard the Van Allen Probes. Figures $3 a-3 c$ show spin-averaged 
differential electron flux in the $2.3,4.5$, and 5.6 MeV energy channels as a function of $L^{*}$ (electron drift shell in the T04 magnetic field model [Tsyganenko and Sitnov, 2005]) between 9 October and 29 November 2012. Similar to the POES observations, a major electron flux decrease is observed on 13 October (purple arrow) during a moderate magnetic storm. However, we did not observe a significant flux reduction in any of the omnidirectional energy channels in Figures 3a-3c on throughout the EMIC event on 11 October (green arrow). Overall, the entire interval can be characterized by a gradual and continuous decrease in flux, interspersed with a sharp step decrease on 1 November, and a similar sharp increase around 15 November 2012.

The most interesting observations from REPT were obtained using pitch angle distributions of these ultrarelativistic electrons. Figures $3 \mathrm{~d}-3 \mathrm{f}$ show electron differential flux as a function of pitch angle in the same $2.3,4.5$, and $5.6 \mathrm{MeV}$ energy channels at $L^{*}=4.5$. In each panel, the flux is normalized by the $90^{\circ}$ pitch angle flux to highlight the changing shape of the pitch angle distributions rather than their changing magnitude. The width of the pitch angle distribution varies during the entire 51 day interval being narrow (i.e., more strongly peaked at $90^{\circ}$ ) at the beginning, then widening, and experiencing fluctuations later in the interval. Also, there is a clear trend toward the distribution being narrower at higher energies. To investigate the effect of EMIC waves on the pitch angle distribution shape, we surveyed EMIC wave occurrence on the ground at two CARISMA stations ( $L \sim 4-4.5$ ). The EMIC wave occurrence seen at these stations is shown in Figure $3 \mathrm{~g}$ and tagged as follows: "No" for days of absent EMIC waves and "Yes" for days where EMIC waves are present. Note that in order to estimate local EMIC wave occurrence on these L-shells, "Yes" is only applied when the EMIC wave power peaks at these L-shells in an attempt to separate local EMIC power from that ducted to the stations from higher L-shells of the CARISMA array. Figure 3 demonstrates an extremely clear correlation and connection between rapid changes in ultrarelativistic pitch angle distributions and the occurrence of EMIC waves. It provides good evidence that EMIC waves can generate bite-outs in flux at low pitch angles, which can last for extended intervals. However, this correlation is not seen in the omnidirectional fluxes (Figures 3a-3c), which indicates that the core of the ultrarelativistic electron distribution was not being significantly affected by EMIC waves during this extended interval.

\section{Electron Pitch Angle Scattering Rates}

We computed bounce-averaged pitch angle diffusion coefficients of electrons due to EMIC waves using a quasilinear approach [e.g., Kennel and Engelmann, 1966; Summers et al., 2007]. Following Meredith et al. [2003], we considered cold multi-ion plasma with the ion composition given by $70 \% \mathrm{H}^{+}, 20 \% \mathrm{He}^{+}$, and $10 \% \mathrm{O}^{+}$. The observed in situ EMIC power spectral density below the $\mathrm{He}^{+}$band was fit by a Gaussian with the following parameters: maximum frequency $0.89 \mathrm{~Hz}\left(0.74 \Omega_{\mathrm{He}}\right)$, bandwidth $0.12\left(0.1 \Omega_{\mathrm{He}}\right) \mathrm{Hz}$, and lower and upper cutoffs $0.65\left(0.54 \Omega_{\mathrm{He}}\right)$ and $1.15\left(0.96 \Omega_{\mathrm{He}}\right) \mathrm{Hz}, \Omega_{\mathrm{He}}$ being the local helium gyrofrequency. Waves were assumed to be field aligned with left-hand circular polarization and confined below the crossover frequency [see Li et al., 2007], within $\pm 13.5^{\circ}$ of the equator. At higher latitudes the waves become oblique, right-hand polarized and do not strongly interact with $\mathrm{MeV}$ electrons. The wave amplitude was calculated as the square root of the integral of the power spectral density divided by frequency, being $2 \mathrm{nT}$. Also, a constant field aligned number density of $150 \mathrm{~cm}^{-3}$ is assumed, derived from the observed Van Allen Probe B spacecraft potential. The computed scattering rates were also MLT averaged assuming EMIC waves were only present over $25 \%$ of the drift orbit.

Figures $4 a-4 c$ show the computed pitch angle diffusion coefficients as a function of equatorial pitch angle for electron energies of $2.3,4.5$, and $7.15 \mathrm{MeV}$, respectively. A particle loss timescale can be estimated using the inverse of the diffusion coefficient. Loss timescales in Figures $4 a-4 c$ lie in the interval of approximately 0.1 to $10 \mathrm{~h}$, depending on the electron energy and pitch angle, being very fast at low pitch angles and significantly slower toward high pitch angles, also increasing significantly with energy from $2.3 \mathrm{MeV}$ to $7.1 \mathrm{MeV}$. Figure 4 demonstrates that with increasing energy, electrons with a larger range of pitch angles are scattered by EMIC waves-narrowing the pitch angle distributions to be more closely confined toward $90^{\circ}$ at higher energies. These calculations clearly show that EMIC waves cause ultrarelativistic ( 2-8 MeV) electron loss, but which is confined to pitch angles below around $45^{\circ}$ (depending on the electron energy) and not affecting the core distribution. Significantly, EMIC pitch angle diffusion rates are very small at large pitch angles, precluding the possibility that EMIC waves can erode the entire ultrarelativistic electron population-specifically they are not capable of scattering the high pitch angle particles into the loss cone. This is in excellent agreement with the REPT instrument energy/pitch angle spectrograms in Figures $4 \mathrm{~d}-4 \mathrm{f}$ showing normalized electron flux as a 


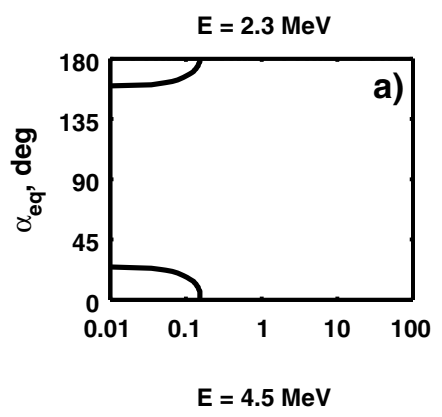

OP77 $L^{*}=4.5 \pm 0.1, R E P T, E=2.3 \pm 0.3 \mathrm{MeV}$
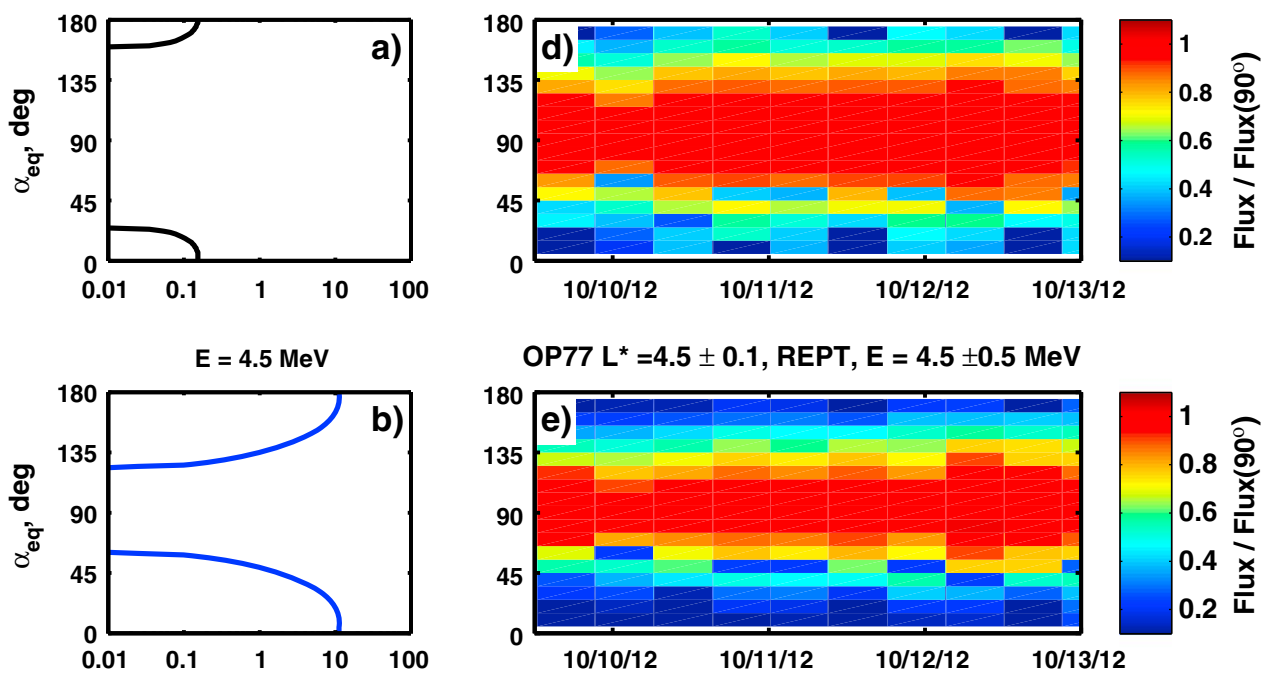

OP77 $L^{*}=4.5 \pm 0.1, R E P T, E=4.5 \pm 0.5 \mathrm{MeV}$
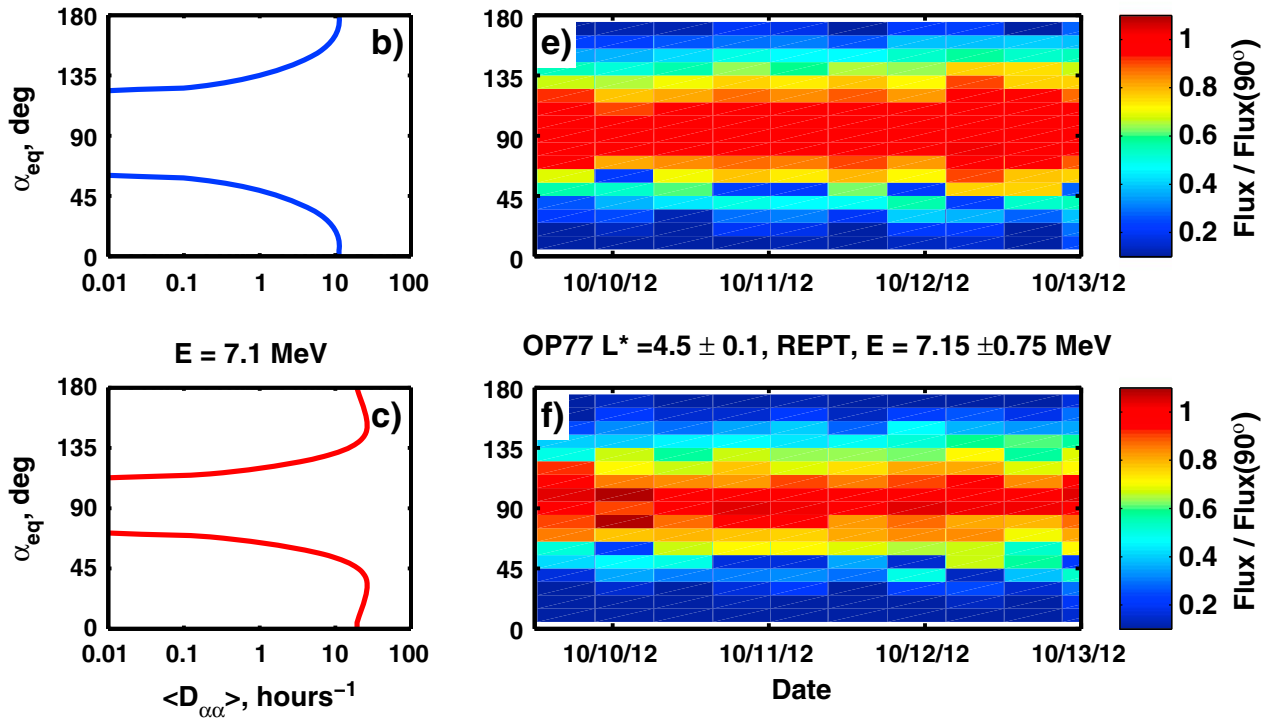

OP77 $L^{*}=4.5 \pm 0.1, R E P T, E=7.15 \pm 0.75 \mathrm{MeV}$

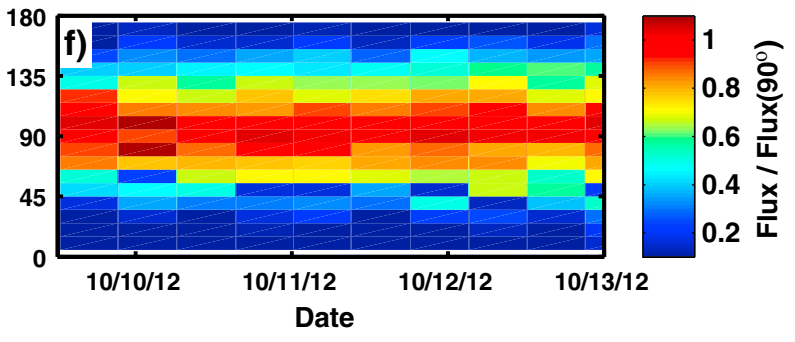

Figure 4. $(a-c)$ Computed pitch angle diffusion coefficients and $(d-f)$ observed normalized electron flux as a function of pitch angle in the 2.3, 4.5, and 7.15 MeV REPT energy channels, respectively, for 9-13 October 2012.

function of pitch angle for 2.3, 4.5, and 7.15 MeV during the times where EMIC waves were present. The dark blue color in this spectrogram corresponds to $<10 \%$ of the locally trapped population and signifies that electrons with these pitch angles are lost.

\section{Discussion and Conclusions}

We investigated the effect of EMIC waves on relativistic and ultrarelativistic electron populations. Using observations from 11 October 2012, during the recovery phase of the geomagnetic storm with minimum Dst on 9 October 2012, we observed precipitation of $30-800 \mathrm{keV}$ protons conjugate to the region of EMIC wave activity - a typical signature of EMIC wave-particle interactions. However, we did not observe precipitation of $>0.7 \mathrm{MeV}$ electrons on POES satellites nor did we observe any decreases of spin-averaged ultrarelativistic electron fluxes on the Van Allen Probes associated with EMIC waves during this period.

Computed pitch angle diffusion coefficients for ultrarelativistic electrons show that EMIC waves with the characteristics observed on 11 October do not affect the core of the electron distribution-consistent with the absence of significant precipitation observed. However, these waves do affect lower pitch angle particles, causing the confinement of fluxes closer to equatorial pitch angles of $\sim 90^{\circ}$, the effect being more pronounced at higher (close to $\sim 8 \mathrm{MeV}$ ) energies. These theoretical results are in excellent agreement with the pitch angle distributions observed by the Van Allen Probe at ultrarelativistic energies.

An important question, which then remains, however, is that since REPT detects the loss of low pitch angle particles due to an EMIC interaction at ultrarelativistic energies, why is this not seen by the POES detector? We can answer this by comparing the flux for the whole $>0.7 \mathrm{MeV}$ population that POES is sensitive to, to the ultrarelativistic flux seen by REPT at $2.3 \mathrm{MeV}$ (first REPT channel) and higher. First, from the AE8 model for active conditions [Vette, 1991] we estimated the ratio between the flux at $>0.7 \mathrm{MeV}$ and $>2.3 \mathrm{MeV}$ to be $\sim 0.1$. 
Second, we calculated that the decline in the REPT flux during active EMIC wave intervals is $\sim 0.15$ of the total flux at $2.3 \mathrm{MeV}$. Since the flux at $>2.3 \mathrm{MeV}$ constitutes only $10 \%$ of the flux at $>0.7 \mathrm{MeV}$, the fraction of precipitating electrons during this event is $\sim 0.01$ of the $>0.7 \mathrm{MeV}$ electron flux detectable by POES. This is below the background precipitating flux (5\%) and hence cannot be resolved by POES. These observations are also consistent with Rodger et al. [2010].

In recent work, Shprits et al. [2013] highlighted the importance of EMIC waves in scattering of ultrarelativistic electrons into the loss cone during the formation of a third radiation belt observed by the Van Allen Probes in September 2012 [Baker et al., 2013]. However, there is no evidence that EMIC waves caused significant loss of either MeV or $>2 \mathrm{MeV}$ electrons during the interval presented here and that any effects on pitch angle distributions were confined to lower pitch angles.

Future modeling and observational efforts are required to address this difference in ultrarelativistic electron response to EMIC waves and to examine the dependence on various wave and plasma parameters in controlling the dynamics of the ultrarelativistic electron population. Since EMIC waves are only effective at scattering lower pitch angle particles, additional transport or diffusion affecting higher pitch angles reaching the core $90^{\circ}$ equatorial pitch angle population (e.g., through interaction with chorus waves-cf. Shprits et al. [2009]) might be needed in order for EMIC waves to significantly deplete the entire pitch angle distribution of ultrarelativistic electrons.

Acknowledgments

We wish to thank the Van Allen Probe $E C T, E F W$, and EMFISIS instrument teams for data. This work is supported in part by the MAARBLE (Monitoring, Analyzing and Assessing Radiation Belt Loss and Energization) consortium. MEU is partly supported by the Canadian Space Agency. IRM is supported by a Discovery Grant from Canadian NSERC. DLT is thankful for funding from NASA (THEMIS contract NAS5-02099 and grant NNX12AJ55G) and the MAARBLE project. The research of K.O. was supported by the NASA Living with a Star Jack Eddy Postdoctoral Fellowship Program, administered by the University Corporation for Atmospheric Research.

The Editor thanks two anonymous reviewers for their assistance in evaluating this paper.

\section{References}

Baker, D. N., et al (2012), The Relativistic Electron-Proton Telescope (REPT) instrument on board the Radiation Belt Storm Probes (RBSP) spacecraft: Characterization of Earth's radiation belt high-energy particle populations, Space Sci. Rev., doi:10.1007/s11214-012-9950-9. Baker, D. N., et al (2013), A long-lived relativistic electron storage ring embedded in Earth's outer Van Allen belt, Sci. Express, 340(6129), 186-190, doi:10.1126/science.1233518.

Cornwall, J. M. (1965), Cyclotron instabilities and electromagnetic emissions in the ultra low frequency and very low frequency ranges, J. Geophys. Res., 70, 61-69.

Evans, D. S., and M. S. Greer (2000), Polar Orbiting Environmental Satellite Space Environment Monitor 2: Instrument Description and Archive Data Documentation, NOAA Tech. Memo. OAR SEC-93, NOAA, Boulder, Colo.

Kennel, C. F., and F. Engelmann (1966), Velocity space diffusion from weak plasma turbulence in a magnetic fields, Phys. Fluids, 9, $2377-2388$.

Kessell, R. L., N. J. Fox, and M. Weiss (2012), The Radiation Belt Storm Probes (RBSP) and Space Weather, Space Sci. Rev., doi:10.1007/s11214012-9953-6.

Kletzing, C. A., et al. (2013), The Electric and Magnetic Field Instrument Suite and Integrated Science (EMFISIS) on RBSP, Space Sci. Rev., doi:10.1007/s11214-013-9993-6.

Li, W., Y. Y. Shprits, and R. M. Thorne (2007), Dynamic evolution of energetic outer zone electrons due to wave-particle interactions during storms, J. Geophys. Res., 112, A10220, doi:10.1029/2007JA012368.

Mann, I. R., et al. (2008), The upgraded CARISMA magnetometer array in the THEMIS era, Space Sci. Rev., 141, 413-451.

Mann, I. R., et al. (2014), Spatial localization and ducting of EMIC waves: Van Allen Probes and ground-based observations, Geophys. Res. Lett., doi:10.1002/2013GL058581, in press.

Meredith, N. P., R. M. Thorne, R. B. Horne, D. Summers, B. J. Fraser, and R. R. Anderson (2003), Statistical analysis of relativistic electron energies for cyclotron resonance with EMIC waves observed on CRRES, J. Geophys. Res., 108(A6), 1250, doi:10.1029/2002JA009700.

Rodger, C. J., M. A. Clilverd, J. C. Green, and M. M. Lam (2010), Use of POES SEM-2 observations to examine radiation belt dynamics and energetic electron precipitation into the atmosphere, J. Geophys. Res., 115, A04202, doi:10.1029/2008JA014023.

Shprits, Y. Y., L. Chen, and R. M. Thorne (2009), Simulations of pitch angle scattering of relativistic electrons with MLT-dependent diffusion coefficients, J. Geophys. Res., 114, A03219, doi:10.1029/2008JA013695.

Shprits, Y. Y., et al. (2013), Unusual stable trapping of the ultrarelativistic electrons in the Van Allen radiation belts, Nature Phys., doi:10.1038/ nphys 2760 .

Spence, H. E., et al. (2013), Science goals and overview of the Energetic Particle, Composition, and Thermal Plasma (ECT) suite on NASA's Radiation Belt Storm Probes (RBSP) mission, Space Sci. Rev., doi:10.1007/s11214-013-0007-5.

Summers, D., and R. M. Thorne (2003), Relativistic electron pitch-angle scattering by electromagnetic ion cyclotron waves during geomagnetic storms, J. Geophys. Res., 108(A4), 1143, doi:10.1029/2002JA009489.

Summers, D., B. Ni, and N. P. Meredith (2007), Timescales for radiation belt electron acceleration and loss due to resonant wave-particle interactions: 1. Theory, J. Geophys. Res., 112, A04206, doi:10.1029/2006JA011801.

Tsyganenko, N. A., and M. I. Sitnov (2005), Modeling the dynamics of the inner magnetosphere during strong geomagnetic storms, J. Geophys. Res., 110, A03208, doi:10.1029/2004JA010798.

Ukhorskiy, A. Y., et al. (2010), Rapid scattering of radiation belt electrons by storm-time EMIC waves, Geophys. Res. Lett., 37, L09101, doi:10.1029/2010GL042906.

Usanova, M. E., I. R. Mann, I. J. Rae, Z. C. Kale, V. Angelopoulos, J. W. Bonnell, K.-H. Glassmeier, H. U. Auster, and H. J. Singer (2008), Multipoint observations of magnetospheric compression-related EMIC Pc1 waves by THEMIS and CARISMA, Geophys. Res. Lett., 35, L17S25, doi:10.1029/2008GL034458.

Usanova, M. E., et al. (2010), Conjugate ground and multisatellite observations of compression-related EMIC Pc1 waves and associated proton precipitation, J. Geophys. Res., 115, A07208, doi:10.1029/2009JA014935.

Vette, J. (1991), The NASA/National Space Science Data Center trapped radiation environment model program (1964-1991), NSSDC Report 91-29, Greenbelt, Md.

Wygant, J. R., et al. (2013), The electric field and waves instruments on the Radiation Belt Storm Probes mission, Space Sci. Rev., 179(1-4), 183-220, doi:10.1007/s11214-013-0013-7. 